\title{
RELATIONS ENTRE RESPIRATION ET POIDS DU CORPS CHEZ LE ZOOPLANCTON D'EAU DOUCE
}

\author{
par R. POURRIOT ${ }^{1}$ et P. CHAMP 1
}

\begin{abstract}
D'après les valeurs ponctuelles ou les formules relevées dans la littérature pour différentes espèces zooplanctoniques, les auteurs établissent une relation moyenne entre métabolisme respiratoire (à $20^{\circ} \mathrm{C}$ ) et poids $(\mathrm{sec})$ du corps, exprimée par l'équation: $\mathrm{R}=0,130 \mathrm{~W}^{0,93}$. Le coefficient $\mathrm{b}(=0,93)$ ainsi calculé est très proche du coefficient moyen $(0,95)$ caractérisant les organismes pœcilothermes de poids compris entre $10^{-6}$ et $10^{-1} \mathrm{~g}$.
\end{abstract}

\section{Relationship between respiration and body weight in zooplankton}

From single values and from equations given in the literature for different species of zooplankton, the authors have developed a mean relationship between respiratory metabolism at $20^{\circ} \mathrm{C}$ and dry body weight ; expressed by the equation : $\mathrm{R}=0.130 \mathrm{~W}^{0.3}$. The exponent $\mathrm{b}(=0.93)$ is very close to the mean exponent $(0.95)$ that is characteristic of poikilotherms in the weight range $10^{-6}$ to $10^{-1} \mathrm{~g}$.

L'intensité du métabolisme respiratoire $(\mathrm{R})$ varie en fonction du poids du corps (W) suivant l'équation classique: $\mathrm{R}=\mathrm{aW}$.

Schoffeniels (1977) constate que les relations exprimant les échanges métaboliques en fonction du poids d'êtres vivants allant des bactéries aux homéothermes, se répartissent en trois zones de poids : 1 . en dessous de $10^{-6} \mathrm{~g}$, 2. entre $10^{-6}$ et $10^{-1} \mathrm{~g}$ et 3 . au-dessus de $10^{-1} \mathrm{~g}$.

Les zones 1 et 3 sont caractérisées par une valeur du coefficient b (pente de la droite de régression pour des coordonnées logarithmiques) proche de 0,75 alors que celle de la zone intermédaire est voisine de 0,95 .

Pour les poecilothermes planctoniques qui se rangent dans cette catégorie de poids, nous avons relevé dans la littérature consultée les relations suivantes entre respiration et poids du corps, après conversion aux même unités $(\mathrm{R}=$ quantité d'oxygène respiré en microgrammes par individu et par heure; $\mathrm{W}=$ poids $\mathrm{sec}$ en microgrammes), à une température égale à (ou voisine de) $20^{\circ} \mathrm{C}$ :

- Daphnia ambigua: R + 0,69 W0,52 (d'après Armitage et Lei 1979)

- Daphnia magna: $\mathrm{R}=0,0211 \mathrm{~W}^{0.816}$ (d'après Kersting et Van der Leeuw-Leegwater 1976),

1. E.N.S., Laboratoire de zoologie, L.A. $\mathrm{n}^{\circ}$ 258, C.N.R.S., 46, rue d'Ulm, 75230 Paris Cedex 05, France. 
1968)

et $\mathrm{R}=0,0096^{3} \mathrm{~W}-6,1.10^{-6} \mathrm{~W}^{2}$ (d'après Schindler

- Simocephalus vetulus : $\mathrm{R}=0,00198 \mathrm{~W}^{0,8}$ (d'après Kryuchkova et Rybak 1971)

$$
\begin{array}{lc}
\mathrm{R}=0,0023 \mathrm{~W}^{1,6575} \text { (jeunes) } & \text { (d'après } \\
\mathrm{R}=0,0113 \mathrm{~W}^{0,825.2} \text { (adultes) } & \text { et Klekowowski } \\
& 1972 \text { ) }
\end{array}
$$

- Leptodora kindtii: $\mathrm{R}=0,0134 \mathrm{~W}^{0,81}$ (d'après Hillbricht-Ilkowska et Karabin 1970)

- Six espèces de Calanoides : $R=\exp _{10}\left(0,095 \mathrm{~W}^{0,417}-1,358\right)$ (d'après Comita 1968 ; la formule originale fait intervenir la température, que l'on a prise égale à $20^{\circ} \mathrm{C}$, et donne la respiration en fonction de la longueur du céphalothorax, que l'on a considérée comme égale à $0,45 \times$ longueur totale du corps; le poids a été estimé en fonction de la longueur du corps d'après les formules citées par Bottrell et coll. 1976).

- Mixodiaptomus laciniatus : $\mathrm{R}=0,01513 \mathrm{~W}^{1,251}$ - 0,1113 (d'après Comita et Comita 1964 ; la formule originale donne la respiration en fonction de la longueur du corps).

- Macrocyclops albidus : $\mathrm{R}=0,0026 \mathrm{~W}^{0,27}$ à $0,0067 \mathrm{~W}^{0,45}$ pour les nauplii, $R=0,0072 \mathrm{~W}^{0,72}$ à $0,0074 \mathrm{~W}^{0,60}$ pour l'ensemble des stades (d'après KLEKOWSKI et SHUSHKINA 1966 ; moyenne générale recalculée d'après les mêmes données par SHushkina, AnIsomov et KlEKowsKi $1968: \mathrm{R}=0,0121 \mathrm{~W}^{0,84}$ ).

D'après les auteurs n'ayant donné que des valeurs ponctuelles de la consommation d'oxygène, les résultats convertis en microgramme d'oxygène par individu et par heure sont les suivants :

- Brachionus calyciflorus :

0,0027 à 0,0070 (d'après Pourriot et Deluzarches 1970)

0,0012 à 0,0032 (d'après auteurs soviétiques cités par PILARSKa 1977)

- Brachionus plicatilis :

0,0019 à 0,0084 selon état physiologique (d'après DooHaN 1973)

- Brachionus urceolaris :

0,0030 à 0,0046 (d'après Pourriot et Deluzarches 1970)

- Keratella valga:

0,0007 (d'après PourRiot et Deluzarches 1970)

- Asplanchna brightwelli :

0,0087 à 0,0157 (d'après Pourriot et Deluzarches 1970)

- Ceriodaphnia reticulata:

0,015 à 0,030 à $22^{\circ} \mathrm{C}$ selon le poids des adultes (d'après GopHEN 1976) 
- Diaptomus siciloides :

0,086 à 0,114 pour adultes à $20^{\circ} \mathrm{C}$ (d'après Comita 1965)

- Cyclops vicinus :

0,141 (d'après BRANDL et FERNANDo 1975) ; la respiration est rapportée par calorie du corps; on a pris pour poids sec du corps $22 \mu \mathrm{g}$, d'après BotTrell et coll. (1976), et pour teneur calorique 5500 cal. par gramme de poids sec

- Mesocyclops leuckarti:

0,0825 (d'après BRANDL et FERnaNdo 1975) ; poids sec : $9 \mu \mathrm{g}$, d'après la formule de Burgis ( $\mathrm{W}=2,2.10^{-8} \mathrm{~L}^{2.82}$; $\mathrm{W}$ en $\mu \mathrm{g}$ et $\mathrm{L}$ en $\mu \mathrm{m}$ ) citée dans Dumont et coll. (1975)

\section{-. Chaoborus punctipennis :}

214 (d'après Comita 1972, pour un poids frais individuel de $10 \mathrm{mg}$ ). Portée en fonction des poids secs des espèces données par les auteurs ou, à défaut, pris dans la littérature, l'ensemble de ces résultats se répartit autour d'une relation moyenne ( $f$ ig. 1) que l'on peut écrire : $\mathrm{R}=0,130 \mathrm{~W}^{0,93}$; l'exposant $\mathrm{b}$ est très voisin de celui cité par ScHoFFENIELS pour les organismes de poids situé dans la zone intermédiaire.

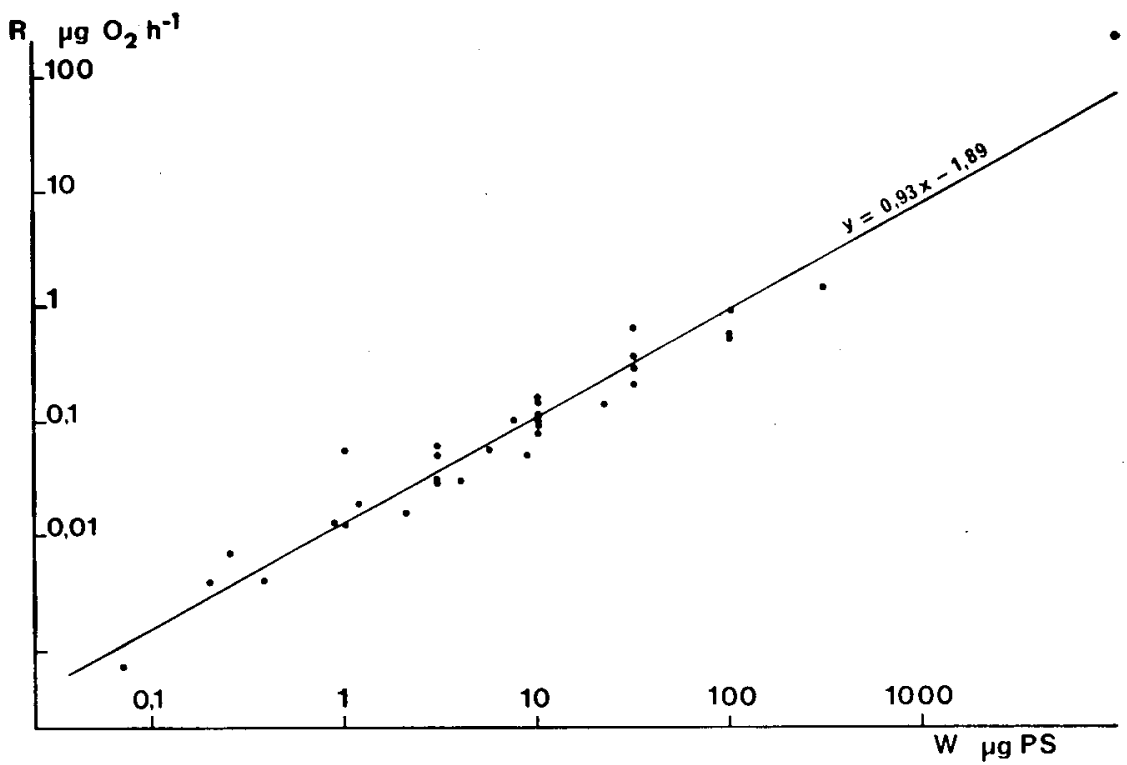

Fig. 1. - Relations respiration - poids du corps ( $\mathrm{PS}=$ poids $\mathrm{sec}$ ) chez les poecilothermes planctoniques: droite de régression en coordonnées logarithmiques $(\mathrm{r}=0,949)$.

Cependant, quelques auteurs ont fourni pour la consommation d'oxy. gène de plusieurs espèces des valeurs qui n'ont pas été intégrées aux précédentes car elles s'en écartaient notablement. Ainsi, selon MosHIRI 
et coll. (1969), des femelles de Leptodora kindtii, ovigères ou non, consomment environ 10 à 15 fois plus d'oxygène que ne l'ont supposé HillbRICHT-IlKowSKa et KaRABiN (1970) d'après une formule générale établie pour les Crustacés. Il est possible que cette espèce qui vit dans les couches d'eaux bien oxygénées, ait des besoins supérieurs à ceux des autres espèces citées ici. Des différences bien plus considérables sont observées pour Eudiaptomus gracilis ; la formule $\mathrm{R}=34,90 \mathrm{~W}^{0,67}$ élablie d'après Kiвby (1971) donne des valeurs environ 1500 fois plus élevées que l'ensemble des autres relations citées : on peut se demander s'il n'y a pas eu erreur dans la mention des unités.

Enfin, les différences de taille entre espèces ne sont évidemment pas les seules causes de variations de la consommation d'oxygène qui dépend aussi des conditions d'acclimatation des animaux (BuiKema 1972 ; Armitage et Lei 1979).

Néanmoins, l'équation calculée ci-dessus permet d'obtenir à partir du poids sec une estimation de la quantié d'oxygène respiré applicable à bon nombre d'espèces du zooplancton d'eau douce.

\section{TRAVAUX CITÉS}

Armitage (K. B.), Lei (C. H.). 1979. - Temperature acclimatization in the filtering rates and oxygen consumption of Daphnia ambigua Scourfield. Comp. Biochem. Physiol., 62 A, 4, 807-812.

Bottrell (H. H.), Ducan (A.), Gliwicz (Z. M.), Grygierek (E.), Herzig (A.), HillBRICHT-IlKowsKa (A.), KuRUSAWA (H.), LaRsSon (P.), WeglensKa (T.). 1976. A review of some problems in zooplankton production studies. Norw. J. Zool., 24, 419-456.

Brandl (Z.), Fernando (C. H.). 1975. - Food consumption and utilization in the two freshwater Cyclopoid Copepods (Mesocyclops edax and Cyclops vicinus). Int. Rev. ges. Hydrobiol., 60, 471-494.

Buikema (A. L. Jr.). 1972. - Oxygen consumption of the cladoceran, Daphnia pulex, as function of body size, light and light acclimation. Comp. Biochem. Physiol., 42 A, 4, 877-888.

Comita (G.W.). 1965. - Oxygen uptake in Diaptomus siciloides Lillj. Limnol. Oceanogr., 10, 466-468.

Comita (G. W.). 1968, - Oxygen consumption in Diaptomus. Limnol. Oceanogr., $13,51-57$.

Comita (G. W.). 1972. - The seasonal zooplankton cycles, production and transformation of energy in Severson Lake, Minnesota. Arch. Hydrobiol., 70, $14-66$.

Comita (G. W.), Comita (J.). 1964. - Oxygen uptake in Mixodiaptomus laciniatus Lill. Mem. Ist. Ital. Idrobiol., 17, 151-166.

Doohan (M.). 1973. - An energy budget for adult Brachionus plicatilis Müller (Rotatoria). Oecologia, 13, 351-362.

Dumont (H. J.), Van De Velde (I.), Dumont (S.). 1975. - The dry weight estimate of biomass in a selection of Cladocera, Copepoda and Rotifera from the plankton, periphyton and benthos of continental waters. Oecologia, 19, 75 . 97. 
GoPHEN (M.). 1976. - Temperature dependence of food intake, ammonia excretion and respiration in Ceriodaphnia reticulata (Jurine). Freshw. Biol., 6, 451-455.

Hillbricht-Illowska (A.), KarabiN (A.). 1970. - An attempt to estimate consumption, respiration and production of Leptodora kindtii (Focke) in field and laboratory experiments. Pol. Arch. Hydrobiol., 17, 81-86.

Ivanova (M. B.), KLEKowSKI (R. Z.). 1972. - Respiratory and filtration rates in Simocephalus vetulus (O.F.M.) (Cladocera) at different pH. Pol. Arch. Hydrobiol., 19, 303-318.

Kersting (K.), Van Der LeeuW-Leegwater (W.). 1976. - Effect of food concentration on the respiration of Daphnia magna. Hydrobiologia, 49, 233-237.

KibBY (H. V.). 1971. - Energetics and population dynamics of Diaptomus gracilis. Ecol. Monogr., 41, 311-327.

Klekowski (R. Z.), Shushkina (E. A.). 1966. - Ernährung, Atmung, Wachstum und Energie. Umformung in Macrocyclops albidus (Jurine). Verh. int. Ver. Limnoi., 16, 399-418.

KRYuchkova (N. M.), RYBaK (V.K.). 1971. - The utilization of food energy by Simocephalus vetulus under different trophic conditions (en russe). Sov. J. Ecoil., 4, 44-50.

Moshiri (G. A.), Cummins (K. W.), Costa (R. R.). 1969. - Respiratory energy expenditure by the predaceous zooplankter Leptodora kindtii (Focke) (Crustacea, Cladocera). Limnol. Oceanogr., 14, 475-484.

Pilarska (J.). 1977. - Ecophysiological studies on Brachionus rubens Ehr. (Rotatoria). b. Production and respiration. Pol. Arch. Hydrobiol., 24, 329-342.

Pourriot (R.), Deluzarches (M.). 1970. - Sur la consommation d'oxygène par les Rotifères. Annls. Limnol., 6, 229-248.

SchindLer (D. W.). 1968. - Feeding, assimilation and respiration rates of Daphnia magna under various environmental conditions and their relation to production estimates. J. Anim. Ecol., 37, 369-385.

Schoffeniels (E.). 1977. - Physiologie des régulations. Masson, Paris.

Shushimina (E. A.), Anisomov (S. I.), KlekowSKi (R. Z.). 1968. - Calculation of production efficiency in plankton Copepods. Pol. Arch. Hydrobiol., 15, 251 . 261. 